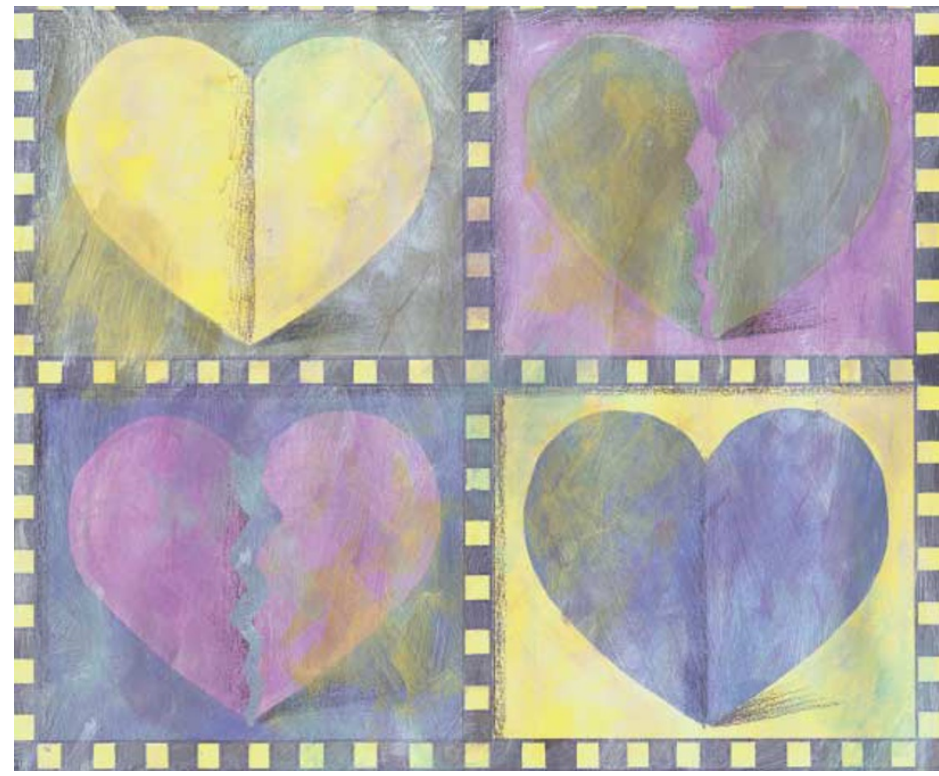

SIGNALLING

\section{Kiss and break up}

Eph receptors and their ephrin ligands are membrane bound, yet they generally mediate repulsive responses. As cell-cell contact must occur to facilitate ligand-receptor interaction, how is this followed by rapid cell repulsion? Two reports in Nature Cell Biology now provide a very attractive answer Eph-ephrin complexes are removed from the cell surface by rapid, localized endocytosis after cell-cell contact.

Zimmer et al. observed the accumulation of internalized ligand-receptor complexes after interactions between EphB2- and ephrinB1expressing cells (which signal bidirectionally). Full-length EphB2 or ephrinB1 clustered at cell-cell contact sites and were trans-endocytosed the receptor was internalized by ligand-expressing cells, and vice versa. This trans-endocytosis required intact Eph and ephrin cytoplasmic tails. For example, truncation of ephrinB1 in the recipient cell impaired internalization into this cell and favoured internalization into the EphB2-expressing stimulator cell. Furthermore, transendocytosis of Eph-ephrin complexes into receptor-expressing cells required Eph kinase activity.

During in vitro co-culture assays of cells expressing ephrinB1 or EphB2, rapid clustering was followed by bidirectional trans-endocytosis and cell retraction. Blocking ephrinB1 endocytosis caused receptor-ligand clusters to grow, be engulfed by the EphB2 cell and induce strong retraction. But truncation of both cytoplasmic tails resulted in strong cell-cell adhesion.
In the other study, Marston et al. used microinjection experiments to study EphB4-ephrinB2 interactions. At regions of ligand-receptor contact, EphB4 phosphorylation was followed by membrane protrusions and ruffles, and the cells subsequently separated. Similar to the results of Zimmer et al., receptor- and ligand-positive internalized vesicles were seen, but in this case, trans-endocytosis into EphB4-expressing cells predominated. The vesicles contained membrane derived from the neighbouring cell surface, but probably didn't arise from clathrin-coat- or caveolae-mediated mechanisms.

So, the localized ruffles and extensions prompted Marston et al. to look at actin-dependent internalization. Indeed, blocking actin polymerization inhibited EphB4 internalization and subsequent cell retraction. Rac-mediated membrane ruffling, in particular, was required for internalization.

Both groups verified the physiological relevance of these results using cell-culture models - of growthcone collapse and venous-arterial boundary delineation - in which they confimed their findings that complexes is needed for cells to kiss and break up.

Katrin Bussell

(4) References and links ORIGINAL RESEARCH PAPERS Zimmer, $\mathrm{M}$. et al. EphB-ephrinB bi-directional endocytosis terminates adhesion allowing contact-mediated repulsion. Nature Cell Biol. 5, 869-878 (2003) | Marston, D. J., Dickinson, S. \& Nobes, C. D. Rac-dependent trans-endocytosis of ephrin-Bs Cell Biol. 5, 879-888 (2003) trans-endocytosis of Eph-ephrin regulates Eph-ephrin contact repulsion. Nature

\section{IN BRIEF}

\section{NUCLEAR TRANSPORT}

The COPI complex functions in nuclear envelope breakdown and is recruited by the nucleoporin Nup153.

Liu, J. et al. Dev. Cell 5, 487-498 (2003)

Even though vesiculation has been largely discounted in having a role in nuclear-envelope breakdown, Liu and colleagues now show that vesicle formation by a COPI-mediated pathway could, in fact, be an important step in this process. They found that the nucleoporin Nup153, which turned out to be crucial for nuclear-envelope breakdown, associates with members of the COPI coatamer complex and is important in directing COPI to the nuclear membrane during mitosis, when nuclear disassembly occurs.

\section{SIGNALLING}

hippo encodes a Ste-20 family protein kinase that restricts cell proliferation and promotes apoptosis in conjunction with salvador and warts.

Wu, S. et al. Cell 114, 445-456 (2003)

The Drosophila Mst ortholog, hippo, restricts growth and cell proliferation and promotes apoptosis.

Harvey, K. F., Pfleger, C. M. \& Hariharan, I. K. Cell 114, 457-467 (2003)

The authors of both of these papers report the characterization of Hippo, a serine/threonine protein kinase of the Ste20 kinase family. Hippo seems to regulate organ size through its ability to regulate cell proliferation and apoptosis. Hippo works in allegiance with the Warts protein kinase and the Salvador tumour suppressor. Phosphorylation of Salvador by Hippo promotes Hippo phosphorylation of Warts. The ternary complex promotes apoptosis by downregulating levels of the caspase antagonist DIAP1, although whether this occurs through a transcriptional or post-translational mechanism is unclear. Loss of Hippo function results in increased levels of the cell-cycle regulator cyclin E, indicating that Hippo restricts cell proliferation by negatively regulating cyclin $\mathrm{E}$.

\section{CYTOSKELETON}

How capping protein binds the barbed end of the actin filament.

Wear, M. A. et al. Curr. Biol. 13, 1531-1537 (2003)

Capping protein $(\mathrm{CP})$ can cap the barbed end of actin filaments, and a 'tentacle' model has previously proposed that the carboxyl termini of the $\alpha$ and $\beta$ subunits of $C P$ are involved in this capping. Now, Wear et al. show that CP does seem to use two independent actin-binding tentacles to cap actin, and that the $\alpha$ tentacle is more important than the $\beta$ tentacle. In addition, as $\mathrm{CP}$ with either tentacle can cap actin, they propose that, rather than contacting one actin monomer at the end of a protofilament, each tentacle contacts two or three monomers and lies at the interface between the two protofilaments. 